\title{
Madres e hijas: ¿Se hereda el modelo de maternidad?
}

Mothers and daughters: is maternity model inherited?

\author{
Almudena de Linos Escario \\ Investigadora, AEDEMO \\ almudenadelinos@gmail.com (ESPAÑA)
}

Recibido: 23.102016

Aceptado: 28.12.2017

\section{RESUMEN}

En el presente trabajo se analiza la posible transferencia del modelo de maternidad entre madres e hijas y los factores de los que puede depender dicha influencia. Se ha llevado a cabo una investigación cualitativa con mujeres con hijos y sus madres y se confirma, en los casos analizados, la hipótesis de partida de que la influencia es positiva. Sin embargo, ello no significa que siempre la estrategia de la hija siga la misma dirección que la de la madre. Se puede deducir, de los casos analizados, que la dirección que adopte la hija estará determinada por dos factores. El primero sería la contemporaneidad de la estrategia de maternidad seguida por las progenitoras, es decir, lo asumible en términos culturales y sociales que la estrategia seguida por la madre sea para la hija. El segundo factor sería la satisfacción de la vivencia en la infancia de las hijas con la estrategia seguida por sus madres. Para que las hijas sigan una estrategia similar a la de sus madres es necesario que dicha estrategia haya sido satisfactoria para la hija en su infancia y que se considere social y culturalmente acorde al pensamiento del momento.

\section{PALABRAS CLAVE}

Maternidad, conciliación, herencia, satisfacción, contemporaneidad.

\begin{abstract}
In this paper, it is analysed the possible transfer of motherhood model between mothers and daughters and the factors that may depend on this influence. It has carried out a qualitative research with women with children and their
\end{abstract}


mothers. In the cases analysed, the hypothesis that the influence is positive it has been confirmed. However, this does not mean that the daughter always take the same direction taken by the mother. The direction that takes this influence is due mainly to two factors. On the one hand, to the contemporaneity of maternity strategy followed by the progenitor, and secondly, to the satisfaction of the experience in childhood daughters with the strategy followed by their mothers. For the daughters to follow a similar strategy of their mothers is necessary that this strategy had been successful for the child in its infancy and that socially and culturally was according to the thinking of the moment.

\section{KEY WORDS}

Maternity, reconciliation, heritage, satisfaction, contemporaneity

\section{INTRODUCCIÓN}

\subsection{Objetivos}

El objetivo del trabajo es analizar hasta qué punto la estrategia seguida por las madres ha influido en sus hijas cuando éstas han tenido que decidir cómo afrontar el dilema de la conciliación de su vida profesional con el cuidado de los hijos, y de qué factores depende dicha influencia.

\subsection{Hipótesis}

La hipótesis que se quiere contrastar con este trabajo es que las madres ejercen una importante influencia en la estrategia que adoptan las hijas. Esta influencia puede ser de dos tipos: por una parte, es posible que las madres hayan ejercido una influencia implícita simplemente con su ejemplo, sin tratar de aconsejar en ninguna dirección. Por otra parte, puede que las madres hayan ejercido una influencia explícita aconsejando a sus hijas, por ejemplo, que lo mejor para sus hijos, y para la familia en general, es tener a la madre el mayor tiempo posible en casa.

Para comprobar esta hipótesis se parte del supuesto de que éste no será el único factor que haya influido. El tipo de empresa en la que trabajan los padres, los horarios, los salarios... muchas variables pueden influir en la estrategia adoptada finalmente. En concreto, la influencia de las madres entraría dentro de un conjunto de variables que construyen el modelo ideal de madre que una mujer se forma a la hora de tener hijos y que suele entrar en conflicto con el conjunto de expectativas e ideales que una mujer que ha estudiado y tiene una profesión, ha desarrollado (Alberdi et al. 2000).

¿Cómo se construye el modelo ideal de madre cuando una mujer tiene o 
se plantea tener hijos? Según González y Jurado, la orientación al empleo de las madres, la influencia de su propia infancia, o la orientación al empleo de los padres serán fundamentales a la hora de construir este modelo (González y Jurado, 2015). Estas cuestiones se tienen en cuenta, aunque en este trabajo el foco se pone en la influencia implícita o explícita que tienen las madres sobre las hijas, que se asume como factor que se añade a otros y no como una explicación alternativa.

\section{ESTADO DE LA CUESTIÓN}

\subsection{Marco teórico}

Como marco teórico principal se utiliza el modelo multinivel de Barbara J. Risman que entiende el género como una estructura social con tres niveles, el nivel individual, el interaccional y el institucional (Risman, 1998). El género, según Risman, debe considerarse una propiedad estructural de la sociedad. No se manifiesta solo en nuestra personalidad, en las reglas culturales o en otras instituciones. El género está fuertemente incrustado como base de la estratificación, diferenciación de oportunidades y constreñimientos, con consecuencias a estos tres niveles.

\section{El modelo multinivel}

El estudio y conceptualización del sexo y el género ha evolucionado considerablemente a lo largo del siglo XX y principios del XXI. Partiendo de las teorías biológicas que explicaban las conductas masculina o femenina en función de aspectos ligados al sexo, se pasó a las teorías psicológicas para las que el género era un rasgo de la personalidad. Cuando los sociólogos comenzaron a estudiar el sexo y el género, se concentraron en el análisis de los procesos socializadores de los niños concluyendo que el rol de género se desarrolla de acuerdo con los estímulos y recompensas que reciben por actuar de acuerdo con los patrones socialmente aceptados (Risman y Davis, 2013). Lopata y Thorne (Citados por Risman y Davis, 2013) llaman la atención sobre el error que supone utilizar explicaciones sobre el rol sexual para explicar diferencias de género, resaltando como nunca se utilizarían diferencias raciales para explicar diferentes oportunidades de las minorías.

A medida que los sociólogos se especializan en el estudio del género surgen dos corrientes teóricas importantes: la que considera que el género no se es, sino que se hace (enmarcadas en las teorías de la interacción) y las nuevas teorías estructuralistas que consideran que las estructuras organizacionales crean las diferencias de oportunidad. Para la teoría de que el género se hace, el género no siempre coincide con el sexo. Aunque uno nace con unas características biológicas concretas, es su manera de actuar la que lo sitúa en un género. Para ser considerado hombre o mujer uno debe comportarse como tal (Risman y Davis, 2013). 
Para los nuevos estructuralistas el género es, sin embargo, un factor neutro, ya que creen demostrar que hombres y mujeres con las mismas oportunidades y limitaciones alcanzan los mismos resultados. Más allá del siglo XX, la conceptualización del género como un sistema que existe fuera del individuo se ha convertido en el nuevo consenso. Muchos científicos sociales han adoptado la idea de que el género no es solo un rasgo de la personalidad, sino que es un sistema social que alienta o restringe los patrones de comportamiento (Risman y Davis, 2013).

Risman parte de una idea de Deaux y Major (1999) que sugieren que el comportamiento depende de la interacción y de las definiciones de sí mismos de los sujetos, las expectativas de los otros y las expectativas culturales propias del contexto. Critica esta perspectiva que, según ella, presta insuficiente atención teórica a la permeabilidad de las desigualdades de género en las organizaciones, por lo que añade que es necesario establecer un vínculo teórico entre las restricciones materiales de lo que podemos hacer con lo que pensamos que somos, y añade al modelo la dimensión institucional (Risman, 1998).

Siguiendo a González y Jurado (2015), que utilizan este modelo teórico en su libro sobre corresponsabilidad "al igual que existe una estructura política o económica, existe una estructura de género que influye en cómo las mujeres y los hombres son socializados desde pequeños, se ven a sí mismos, interactúan entre ellos y cómo las instituciones construyen diferencias de género" (González y Jurado, 2015: 15). Entre los elementos que influyen en la división del trabajo y en la construcción de la maternidad y paternidad estarían los factores individuales (socialización, habitus, situación y experiencia); los factores relacionales/ pareja (interacción) que se concretan en la negociación o no de las prácticas y acuerdo o desacuerdo en los valores; y los factores culturales e institucionales, es decir, el conjunto de normas sociales, las políticas familiares y el mercado de trabajo.

En el plano individual, siguiendo el esquema elaborado por González y Jurado (2015: 16) sobre el modelo multinivel, encontramos las expectativas hacia la carrera profesional, la dedicación al trabajo, el deseo de formar una familia o el tipo de familia que se desea formar, así como los valores de igualdad, justicia o amor que tiene la persona. En el mismo nivel encontramos las referencias de comparación y las representaciones sociales del individuo en la pareja, la familia, los amigos y, en lo que atañe a este trabajo, los padres y en concreto, la madre. En este nivel individual se encuentra el margen de libertad que proporciona al individuo los recursos materiales, sociales o emocionales de que disponga. En el nivel de la interacción encontramos los ideales que se forman en la hija debido a la interacción con la madre como referencia o representación social de lo que es y debe ser una madre y el rol que debe desempeñar. Y el nivel institucional, como se ha comentado, sería el marco cultural y legal en el que estas mujeres se desenvuelven.

En este sentido, muchas madres se enfrentan hoy en día con un importante dilema en el plano individual entre dedicar su tiempo y recursos al trabajo remunerado y su compromiso con la familia (Alberdi et al. 2000). Esta contradicción 
es parte de lo que Hays (Hays, 1998) denomina las contradicciones culturales de la maternidad. Para Hays, estas contradicciones se originan en la confrontación, por una parte, entre un modelo tradicional que se basa en la concepción histórica de que el cuidado de los hijos es una responsabilidad de las mujeres, que el hábitat natural de las mujeres es el privado del hogar y el del hombre es el público del trabajo remunerado, con, por la otra, la concepción moderna del trabajo remunerado y sus exigencias derivadas de la competitividad del mercado, o con la necesidad de triunfar en ambos campos.

Para Hays, la 'maternidad intensiva' significa vivir la maternidad como la tarea más importante de la vida de las madres y principalmente responsabilidad de ellas, en quienes recae esta labor por ser las mejor preparadas para ello. Estas mujeres a menudo utilizan su sueldo para elevar el estatus económico de la familia, para vacaciones, caprichos o simplemente para poder ahorrar. Por ejemplo, las madres americanas contemporáneas, desde su punto de vista, creen que esta dedicación intensiva en energía, tiempo y recursos materiales es la mejor opción para la correcta evolución y desarrollo de los hijos. Según Hays, este modelo de maternidad constituye la ideología dominante en la sociedad americana de su tiempo, finales del SXX aunque otros estudios no lo han confirmado (González y Jurado, 2015).

No todas las mujeres se adscriben al modelo de la maternidad intensiva. La "madres cuidadoras corresponsables" (González y Jurado, 2015:78) no se consideran las cuidadoras principales, se resisten a la idea de la maternidad intensiva e intentan mantener un equilibrio entre el cuidado de los hijos y su propia vida. Estas mujeres comparten las tareas con "padres comprometidos" (González y Jurado, 2015:121) y mantienen un reparto equitativo de las tareas domésticas resultado de una ideología de género igualitaria que ya se practicaba con anterioridad a la llegada del primer hijo. Para estas mujeres, el empleo sigue siendo importante una vez que son madres y disponer de un padre accesible les permite no tener que realizar adaptaciones laborables que otras mujeres si tienen que adoptar.

Analizaremos en este trabajo si las madres que han optado, a priori de forma voluntaria, por la reducción del tiempo dedicado al trabajo remunerado para el cuidado de los hijos se distancian o no de este modelo de madres corresponsables y padres comprometidos, y las razones de este distanciamiento.

\subsection{Contexto sociodemográfico}

Las protagonistas de este trabajo podrían representar la dupla de generaciones de lo que Garrido denomina "Las dos biografías de la mujer en España" (Citado por Garrido, en Garrido 1998). Para Garrido, la división intergeneracional se produce entre 1950 y 1955. Las representantes de la segunda biografía, las "nuevas españolas", son mujeres con estudios universitarios que buscan una consolidación laboral, principalmente en el sector público, y posteriormente tienen hijos. Por el contrario, las pertenecientes a la primera biografía, asociadas 
con las "familias tradicionales", algunas de ellas nacidas en la posguerra, en su mayor parte solo cuentan con estudios primarios o no tienen estudios, y la mitad de ellas nunca han trabajado.

Entre las mujeres que pertenecen a las generaciones que vivieron la primera biografía, la mayoría de las que no trabajaban abandonaron el trabajo antes de los 30 años, con independencia de la edad, siguiendo la pauta tradicional de dejar de trabajar al contraer matrimonio. Por el contrario, las 'nuevas españolas' (Garrido, 1998: 26), con índices de ocupabilidad que llegan al 79\%, continúan trabajando en su inmensa mayoría cuando se casan (solo un 11\% se dedican únicamente al hogar).

En la situación actual, tras ocho años de crisis económica, se ha truncado el ritmo de incorporación de la mujer al empleo que se dio durante la época de bonanza. Mientras que los primeros años de la crisis fueron relativamente positivos para la mujer por estar mayoritariamente empleadas en el sector público y en el sector servicios, la segunda parte de la crisis las afectó de lleno (Castaño, 2015). A partir de los años 90, en Europa, los diferentes 'regímenes de género' (PfauEffinger citado por Castaño, 2015) se van unificando hacia una mayor presencia laboral de las mujeres gracias a su mayor nivel educativo, evolucionando desde el típico modelo patriarcal de marido sustentador y madre ama de casa, hacia el modelo de familia de doble ingresos apoyado, a su vez, por el desarrollo del Estado de bienestar que amplía las licencias de maternidad/paternidad y apoya los servicios públicos. En España se han producido importantes cambios en el modelo de familia que ha visto como crecían los hogares con formaciones diferentes a la familia tradicional de padre, madre e hijos. El modelo tradicional de varón como único sustentador ya no es el más habitual. Este tipo de hogares que representaba el $48 \%$ en 1999, pasa al $27 \%$ en 2014, mientras que las parejas de doble ingreso pasan en los mismos años del 43 al 54\%, si bien tuvieron su punto más alto en 2007 con el 61\% (González y Jurado, 2015). En este nuevo escenario "las mujeres experimentan un cambio en sus aspiraciones respecto al trabajo y a su posición en la familia, y aceptan mucho peor que antes los roles tradicionales de género" (Castaño, 2015: 28). Sin embargo, "la presencia de una pareja implica una menor tasa de ocupación de las mujeres" siendo al contrario en los hombres (Castaño, 2015: 208) al igual que la presencia de hijos pequeños (menores de 3 años). Conviene matizar que, aunque la presencia de una pareja y de hijos pequeños afecta más a la mujer que al hombre en el plano laboral, las estrategias de las mujeres han experimentado un importante cambio en los últimos años. Si en 1999 la tasa de empleo de las mujeres que viven en pareja con un hijo menor de 3 años era del 56\% en 2014 ésta se eleva hasta el 70\% (González y Jurado, 2015). 


\section{METODOLOGÍA}

\subsection{Una investigación cualitativa}

Se ha optado por una aproximación metodológica cualitativa sobre la base de grupos de discusión frente a otras metodologías por parecer la más idónea en un estadio inicial de conocimiento del campo objeto de estudio, como es el presente, ya que no se ha encontrado bibliografía sobre el análisis intergeneracional del ideal de maternidad. Mientras la investigación cuantitativa requiere de un conocimiento previo relativamente amplio que permita el propio diseño de la investigación, la investigación cualitativa es más abierta y flexible, y permite "captar lo imprevisto" e ir adaptándose según se avanza en la investigación (Corbetta, 2003). Este trabajo se enfoca como una primera toma de contacto, una primera aproximación a la investigación sobre el traspaso de modelos entre madres e hijas y, como tal inicio, persigue, por el momento, la comprensión del fenómeno, la riqueza de los datos y su profundidad, y deja para fases posteriores tanto la ampliación de la base de la investigación y casos, como su posible cuantificación.

Las dos técnicas que se emplean habitualmente en investigación cualitativa y que podrían haberse empleado en este trabajo son el grupo de discusión y la entrevista en profundidad. Como dice el profesor Callejo "mientras que el uso de la entrevista apenas necesita ser justificado, el uso del grupo de discusión suele exigir una explicación" (Callejo, 2001: 37). Según este mismo autor, existe un consenso en algunos autores sobre la capacidad del grupo para permitir que los individuos intercambien posiciones sin tener que defenderse ya que se encuentran entre iguales; el ambiente creado en el grupo de discusión tiende a favorecer que las personas hablen más abiertamente de algunas temáticas al sentirse arropadas por un grupo de personas con similares circunstancias (Callejo, 2001). Asimismo, el grupo de discusión, al fomentar le conversación entre los participantes, produce una mayor riqueza de datos, dado que unos participantes inspiran a otros y la conversación puede discurrir por temas que previamente el investigador podría no haber tenido en cuenta, aspecto esencial en las fases iniciales de una investigación. Sin embargo, el grupo de discusión tiene la desventaja, frente a la entrevista en profundidad, de dificultar el análisis particular o de individuos concretos, ya que las conversaciones se entremezclan. La técnica empleada ha resultado, según se esperaba, adecuada al objetivo del trabajo, al crearse un intenso ambiente de complicidad y conexión entre las mujeres, que se han abierto y han relatado experiencias ricas y emocionales. Unas y otras han observado cómo eran diferentes las circunstancias y contextos entre ellas, pero como, a la vez, compartían muchos de los sentimientos, frustraciones y problemas a los que cada una se había enfrentado o se enfrenta actualmente.

Esta investigación se ha llevado a cabo sobre la base de dos grupos de discusión con 7 participantes cada uno (es decir, siete madres y sus siete hijas) de dos horas y media de duración cada uno. En primer lugar se llevó a cabo el grupo de las madres y posteriormente el de las hijas. Se optó por esta secuencia para 
recoger sus relatos de manera completamente espontánea, ya que de las madres no teníamos ningún tipo de información previa; la única condición para participar en la investigación era que fueran las madres de las mujeres que queríamos estudiar.

Las reuniones han tenido lugar en Madrid, el día 29 de marzo de 2016. La moderadora de ambos grupos ha sido la autora de este trabajo.

\subsection{MUESTRA}

Uno de los grupos de discusión estaba formado por siete mujeres entre 33 y 41 años de edad, con hijos (entre uno y tres hijos de 1 a 10 años; dos de ellas embarazadas del segundo hijo) de las que seis tenían estudios superiores y otra, secundaria; todas ellas con empleos remunerados, dos de las cuales trabajaban en la sanidad pública y el resto en empresas privadas, una de ellas en la empresa familiar. Todas habían solicitado de forma voluntaria la reducción de jornada en al menos el 33\% (siendo la mayoría del 50\%), todos sus maridos o parejas estaban activos laboralmente, con horarios variables, algunos con extensas jornadas laborales y otros con jornadas más compatibles con el cuidado de los hijos.

El otro grupo de discusión estaba formado por las madres de las mujeres del grupo anterior a las que no se les pidió ningún tipo de característica para participar en la investigación, sino ser las madres del grupo anterior. A lo largo de la investigación se recogió su perfil sociodemográfico. Estas mujeres tenían entre 55 y 67 años; de las siete, cinco estaban casadas, una divorciada y otra era viuda. Tenían entre uno y seis hijos, y dos de ellas tenían nietos. Todas ellas habían tenido algún empleo en algún momento de su vida, incluso después de casadas y con hijos.

\section{¿Por qué se ha elegido este perfil?}

En particular se ha buscado un perfil que permitiera aislar, en la medida de lo posible, la variable objeto de análisis, esto es la posible influencia materna en la estrategia seguida por las mujeres a la hora de cuidar a los hijos. Por este motivo se ha seleccionado a mujeres con una carrera profesional activa anterior a ser madres, un nivel de educación que haya significado un esfuerzo y la dedicación de unos recursos significativos de manera que no sea la falta de incentivos profesionales lo que les haya motivado a ralentizar su carrera profesional, y por último una situación de reducción de jornada remunerada voluntaria, de manera que no haya sido una decisión forzada por la empresa o institución. En el caso de las madres, como se ha mencionado, únicamente era necesario que fueran las madres de estas hijas, sin ninguna característica específica. 


\section{ANÁLISIS DE LOS CONTENIDOS}

\subsection{Resultados de la investigación}

La exposición de los resultados de la investigación se inicia con un análisis del contexto en el que madres e hijas llegan a la maternidad. Se analiza el significado, para las participantes de ambas generaciones, de los estudios, el trabajo remunerado, la familia y las circunstancias en las que se desarrollan sus estrategias. Tras el estudio desde un punto de vista generacional, se analiza y compara, caso por caso, la estrategia seguida por las madres y sus hijas.

\subsection{Expectativas de vida}

\subsubsection{Las madres}

Salvo excepciones, los estudios no fueron la prioridad en las mujeres de este estudio. Antes que estudiar, su prioridad era ponerse a trabajar, y, antes incluso que trabajar, su prioridad era casarse. Trabajar y casarse eran los dos pasos principales en su camino hacia la independencia. Independencia y liberación en gran parte del yugo paterno. Tener algo de dinero propio, salir de casa y vivir otro ambiente diferente al familiar, suponía ir despegándose de la vigilancia del padre, figura de gran autoridad. El padre decidía si las mujeres estudiaban o no, si trabajan, sus horarios, su ocio... Primero el trabajo, pero fundamentalmente casarse, significaba para ellas la liberación definitiva del padre. Para muchas, además, significaba tener hijos, algo que colmaba sus máximas expectativas de vida.

\section{trella). \\ "Todos queríamos independizarnos, trabajar. Tener nuestro dinero" (Es- \\ "Era la idea de independencia, de tener tu casa, tus hijos" (Maisa).}

Encontramos dos perfiles de mujeres en el grupo estudiado. De nuestras siete participantes, cuatro no le daban, en esos primeros años, una gran importancia al trabajo remunerado. No tenían la aspiración de completar una carrera profesional. El empleo era una situación transitoria antes de casarse y tener hijos. Representan el modelo tradicional de cuidadoras.

"Ya dejabas de sufrir, tenías algo más seguro. Yo no tenía nada. ¡Y decía cuando me case voy a tener mi casa, mi marido... mis hijos!” (Maite).

"Yo más que casarme lo que quería era tener hijos. ¡Tenía una ilusión enorme!" (Esperanza).

Sin embargo, otras tres mujeres, con diferente nivel educativo y en algún caso más jóvenes, si expresan una mayor aspiración profesional representando al modelo algo más evolucionado de cuidadora principal, no exclusiva. 
De ellas una, aunque no es su ideal, tiene que dejar de trabajar para dedicarse a su familia porque a su marido lo trasladan de ciudad y es ella, con un empleo con elevada proyección social y para ella muy satisfactorio y siendo la que más dinero gana de la pareja, la que renuncia a su trabajo. "Yo cuando a mi marido le trasladaron le sugerí que como yo ganaba más nos quedáramos, pero eso era impensable" (Estrella). Las otras dos mujeres, una universitaria y la otra no, expresan una gran firmeza en su deseo de trabajar toda su vida. "Yo tenía clarísimo que quería seguir trabajando" (Reina). Una de ellas se mantuvo activa con empleos a tiempo parcial y la otra con empleos a tiempo completo, una elevada responsabilidad y viajes frecuentes.

"Si no hubiera encontrado a mi marido no me habría casado, no era mi meta. Mis hermanos estudiaban y habían acabado la carrera, pues yo lo mismo" (Reina).

"Yo tenía un trabajo en una multinacional muy importante" (Silvia).

Estas experiencias reflejan bien la realidad de estas generaciones de mujeres, nacidas alrededor de 1955, que acceden al trabajo con la llegada de la democracia, una época en la que era excepcional que la mujer trabajara, mucho menos si estaba casada y tenía hijos.

"Para empezar te preguntaban si tenías novio, yo decía que no, por supuesto. Era muy masculino, muy competitivo. (...) Cuando me quedé embarazada me miraban mal en la asesoría en la que trabajaba. Un amigo de mi hermano me contrató embarazada de 6 meses, fue inesperadísimo, tenía un horario por la tarde que me permitía ocuparme de mi hijo por la mañana” (Reina).

Los negocios familiares, en general los del marido, han significado para muchas de ellas (en esta muestra tres de siete) una buena alternativa para trabajar a la vez que ocuparse de las tareas domésticas y de los hijos suponiendo, a la vez, una ayuda para el negocio, ya que, por lo general, no cobraban un sueldo ni estaban dadas de alta en la seguridad social.

Todas ellas manifiestan hoy que el ideal es compatibilizar el empleo con el cuidado de los hijos pero, frente a las dificultades de conciliación, la mayoría (todas salvo un caso) dio prioridad en su día al cuidado de los niños, incluso si ello significaba tener que abandonar sus empleos por completo. De las parejas solo esperaban, como máximo, que ayudasen en las tareas logísticas de recoger a los hijos en el colegio, en algún caso ayuda con los baños y las cenas, o no nada en absoluto..

"Estaba con mi hijo toda la mañana, le arreglaba, se lo llevaba a mi madre (...) y tenía un horario de tardes que me permitía cuidar de mi hijo por la mañana. Mi marido le buscaba a las seis en casa de mi madre. Mi madre me ayudaba muchísimo, pero con el segundo hijo, al año y medio, ya los empecé a llevar a la guardería" (Reina)

"Yo dejé el trabajo y me dediqué a ellos. Cuando mi marido puso la tienda, mi hijo más mediano tenía 3 años y tuve que echar una mano, pero no cogían 
en el colegio hasta los 4 años. Conseguí que me lo cogieran en un colegio y durante ese rato yo ayudaba en la tienda a mi marido, y cuando salían del cole recogía al niño. Pero al final, a partir de ese momento, ya era más cuidar de los niños que trabajar, ...además tenía a mi suegra ciega en casa, corrías mucho. Cuando tuve al tercer niño, ya era un poco más relajado, iba menos a trabajar" (M $\mathrm{M}^{\mathrm{a}}$ Luisa)

"Contemplé la posibilidad de contratar a una chica, la tuve de prueba y era un desastre, fue imposible. No me fiaba. Tomé entonces la decisión de ser madre, ocuparme de mis hijos y así he estado durante muchos años" (Estrella)

"Cuando me casé ya no tenía opción, mi marido ya tenía sus negocios, yo no tenía madre, mi suegra era muy mayor y ya tenía el primer hijo. Lo tenía claro que tenía que asumirlo. Pedí la cuenta en mi empresa, me indemnizaron y empecé con este nuevo camino." (M $\left.\mathrm{M}^{\mathrm{a}} \mathrm{José}\right)$

"No quise tener tantos, aquello me vino regalado, pero realmente me habría gustado (trabajar). Mi marido tampoco era partidario de que trabajara... es verdad que me planteaba meterlos en la guardería, pero era mi sueldo y mucho más" (Esperanza)

"Yo trabajaba, ganaba muy poquito, pero había que pagar el piso... Mi marido estuvo en el paro un año y tenía que salir a trabajar. Había que mantener a tres niños (...) No te ayudaba nadie y al final conciliar todo era algo que ni te planteabas" (Maite)

"Yo estuve con mi hija 6 mesecitos y en seguida la llevé a la guardería, a una que estaba al lado de casa" (Silvia)

\subsubsection{Las hijas}

Las hijas, todas con estudios superiores (seis) o medios (una) daban por hecho que seguirían empleadas cuando tuvieran hijos. Ninguna de ellas se planteó dejar por completo sus empleos. Algunas intentaron, con los primeros hijos, mantener la jornada a tiempo completo y otras, sin embargo, ya planeaban desde el principio reducirse la jornada durante un tiempo, más o menos indeterminado, hasta que los hijos "fueran mayores". Para la mayoría de ellas, la llegada de los hijos supone una importante quiebra en sus expectativas profesionales, el enfrentamiento a la "cruda realidad", bien porque no consiguen conciliar empleo e hijos, bien porque esperaban que la reducción de jornada no penalizara sus trayectorias profesionales.

“Yo pensaba que podría con ello (...), nos organizábamos bastante bien (...) pero poco a poco iba reduciendo sin darme cuenta. Cuando tuve el segundo decidí que la reducción de jornada era la mejor opción. Trabajo mucho desde casa (...) pero aun así no te da para tanto, es muy complicado. (Lara)"

"Si, lo intenté (seguir trabajando), pero no siempre es lo que tú quieres (Teresa)" 
La mayoría de ellas muestra una gran disposición hacia la maternidad. Casi todas ellas querían tener hijos, era una expectativa importante en sus vidas.

\section{"Yo siempre he querido tener niños" (María)}

En este sentido, todas consideran que cuidar de ellos es una experiencia muy importante en sus vidas, y que esta tarea les corresponde fundamentalmente a ellas, sobre todo en los primeros meses/primer año. En relación con la decisión sobre la reducción de jornada, se desprende de sus discursos que, aunque en algunos casos el tema se discutió en pareja, la decisión última era de ellas, era una decisión personal. En ningún caso, se menciona la posibilidad de que hubiera sido el padre el que se cogiera la reducción de jornada. La decisión sobre la reducción de jornada gira en torno a si es posible compatibilizar los horarios de trabajo de ellas con el cuidado de los niños. En algún caso se menciona la posibilidad de que sus madres les ayuden como vía para evitar completamente la reducción de jornada. Esta reducción ha ido, en estos casos, más allá de la necesaria para mantener la lactancia natural, ante la que tenemos variedad de posturas (desde las críticas por pensar que supone una esclavitud para la madre, hasta la profesional sanitaria que la defiende a ultranza, pero todas reconocen la enorme presión social que existe actualmente en pro de la lactancia natural).

"En mi caso, opinamos los dos, pero sí que es verdad que soy yo la que lo decido como soy yo la que voy a coger la reducción. Con su apoyo obviamente. Si yo me quisiera ampliar la jornada me diría, vale. Estoy convencida de que en eso soy yo la que decido". (Irene)

Las mujeres participantes manifiestan un deseo de extender la jornada reducida lo más posible, pero nunca si supone la pérdida del empleo. Mantener un contacto con el mundo laboral es muy importante para todas ellas. El empleo, más allá de la importancia económica que tiene en el hogar, supone una cierta dignidad, un contacto con el mundo adulto y un reconocimiento externo. Todas consideran que actualmente "está muy mal visto" dejar el empleo.

Pero la jornada reducida ha significado para todas ellas (salvo en los dos casos de las enfermeras que trabajan en la sanidad pública) un frenazo en su carrera profesional que, incluso en algún caso, consideran perdida para siempre. Esto se vive con un profundo sentimiento de frustración que tienden a justificar refugiándose en la idea de que al menos se están ocupando de sus hijos, algo prioritario para ellas.

"Yo vivía antes en Londres y cuando decidí que quería tener hijos, volví a España. También con la intención de ayudar un poco en la fábrica. Tenía un puesto increíble, todo de color de rosa y vine aquí y no es que diga que la jorobé porque los niños... pero ha cambiado totalmente, yo quería seguir con la empresa, hacerme cargo de todo, pero con el segundo... y la empresa no va como querría, y tampoco le podía meter tantas horas en la guardería, es la pescadilla que se muerde la cola, pero vamos no di abasto. No me arrepiento, porque mis niños son lo mejor que tengo, pero cambió bastante mi vida, es 
complicado organizarse, aquí estoy, esperando que empiece el colegio para reanudar un poco el trabajo...Lo volvería a hacer, pero mi vida no tiene nada que ver con lo que había planeado (...) Luego compensa, pero estoy estancada ahora mismo. No puedo hacer lo que quisiera... disfrutar de mis hijos eso sí... que es lo más importante (Lara)".

"Cuando te incorporas intentas mantener el ritmo. Te das cuenta de que no llegas al ritmo, que no te da tiempo a nada. Ni estaba bien en el trabajo ni pasaba tiempo con mi hijo. Me reduje un poco la jornada, un par de horas, y al tiempo me la bajé a la mitad. Dije se acabó. Las expectativas que tenia de progresar de llegar a una cierta categoría un sueldo y luego tener hijos. No podía. Mis expectativas se truncaron ya en el embarazo, sin quererlo pero.... Feliz ahora. En su momento me pareció un drama entre comillas, pero ahora lo veo con otra perspectiva. Me veo todavía muy joven, mi hijo se está haciendo mayor. Creo que tengo una segunda oportunidad a ver si me subo al tren. Está por ver... (Irene)."

"Creía que mis compañeros tenían que respetar y adaptarse a mi maternidad. Reduje mi jornada porque quería dedicarme a mis hijos (...) Me echaron un poco para atrás en todos los proyectos, con el segundo todavía fue peor y no te cuento con el tercero... (Marta)"

La imposibilidad de conciliar trabajo e hijos se focaliza, además de diversas circunstancias (distancia física con los abuelos, tipo de actividad tanto de ellas, como de sus parejas o, incluso, la presión social), en los horarios de los trabajos, no sólo de ellas, sino también de sus parejas, que justifican por la precariedad laboral y la necesidad de proteger los empleos de los hombres. Salvo el caso de algún funcionario público, con mayor seguridad laboral y mejores horarios, la crisis económica se ha sentido en estos hogares en los que la vuelta a la actividad laboral del hombre ha supuesto un gran alivio.

"A él no le pide el cuerpo tanto, estar en casa, tiene más trabajo, está más liado... Ha pasado una temporada bastante parado, mucho tiempo libre en casa (...), y ahora está desatado. Ahora va de 8 a 18 de la tarde y está encantado de la vida" (María)

"Mi marido tiene jornada completa, es profesor, y era imposible" (Raquel)

"Probé un mes (la jornada completa). Yo tengo turno de tarde y mi marido trabaja en banca y es jefe de departamento, así que tiene un horario un poco.... Igual llegaba a casa a las 9 de la noche" (...) "Creo que socialmente, aunque haya excepciones, todavía está demasiado arraigado que el hombre trabaja más, está mal visto que sea el hombre (el que se coja la reducción de jornada), aunque he visto casos, pero la mayoría es la mujer la que más tira de ahí... (Teresa)

Su ideal, que coincide en general con el ideal de sus propias madres, pasa por ser unas madres presentes durante el tiempo que los niños no están en la escuela. Consideran que su responsabilidad es acompañar a los niños desde que salen del colegio hasta la hora de dormir. Esas primeras horas de la tarde, las tareas extraescolares, los deberes, la merienda es una tarea que muy excepcionalmente comparten con sus parejas. Ya por la noche, baños y cenas es algo en 
lo que sí esperan ayuda del padre y si ésta no se produce es cuando se producen las tensiones. En el caso de las mujeres que tienen turnos de tarde o noche, la situación se compensa por tener algunos días libres.

"Yo echo de menos que mi marido pase más tiempo con la niña cuando llega de trabajar, que asuma su responsabilidad. Llega y se planta una hora con el ordenador, o se ducha y mira la tele. Oye! Que tienes una hija, hazle caso... Luego me dice que la lleve a la guarde para tener más tiempo para mi, para desconectar, pero no la tendría que llevar a otra parte si él le hace el caso que le corresponde. Con que él este en casa me es suficiente, y yo me iría al gimnasio" (María)

Todas ellas han primado el trabajo de sus parejas sobre el suyo propio y reconocen que actualmente la sociedad no está preparada para que los hombres sean los que soliciten la reducción de jornada (incluso las licencias por maternidad). Sin embargo, tampoco ellas parecen estarlo. Todas reconocen que, si la reducción de jornada la pidiera su pareja y ellas tuvieran que trabajar a tiempo completo y se "perdieran" esos momentos con sus hijos, lo vivirían con una gran tristeza. El ideal sería poder compaginar ambas cosas, que los horarios de ambos no fueran tan extensivos.

"Mi marido no pudo cogerse los días de maternidad porque estaba mal visto, y no solo por opinión, pero a nivel represalias no sabía tampoco el alcance. Se sentía desprotegido" [Aunque la ley se lo permitía, 2013] (Ana)

\subsubsection{Una perspectiva diferente: Las madres miran hacia atrás, las hijas viven el ahora}

Conviene tener en cuenta que las madres hablan hoy de su vida desde la perspectiva que da los años, mientras que las hijas hablan de lo que están viviendo actualmente, algunas de ellas iniciando el camino, con un hijo y otro en camino, y otras ya con el número de hijos que desean, encarando ya el final de la primera etapa infantil. Este diferente plano desde el que hablan podría dar lugar a diferencias en sus discursos provenientes no de las diferencias de sus ideales, sino de diferencias en la perspectiva con la que se enfrentan a la maternidad.

En primer lugar, en las madres observamos como la vida les ha ido llevando por caminos muchas veces inesperados, no planeados, a los que han tenido que adaptarse, y que hace que transmitan a sus hijas la necesidad de la cautela, de protegerse antes los avatares de la vida.

En segundo lugar, conviene tener en cuenta el momento de profundo cambio sociopolítico por el que estas mujeres (las madres) atravesaron. Casi todas ellas (salvo un caso considerablemente más joven y otro que por estudiar una carrera sale al mundo laboral más tarde) son mujeres que viven el paso de la dictadura a la democracia justo en el momento en que son madres y, por tanto, vivieron los cambios sociales más profundos que se ha vivido en España en los últimos tiempos (divorcio, anticoncepción, aborto etc.). 
Estos dos hechos son muy significativos, ya que se ve en ellas una evolución importante como mujeres, un cambio de perspectiva sobre lo que una mujer debía o no hacer en la vida. En ciertos aspectos se refieren a su pasado, a las circunstancias en las que vivieron, con cierta perplejidad y, aunque no asumen completamente todas las tendencias actuales, adoptan muchas veces una actitud más avanzada de lo que se podría esperar de su generación. En general, tratan de mantener lo que consideran lo mejor de su época y modificar lo que consideran peor.

Así, mientras que, por una parte, la mayoría defiende la conveniencia de que las madres se ocupen activamente del cuidado de los niños, en general todas aconsejan a sus hijas que no cometan el error que ellas cometieron de no trabajar. La situación que viven sus hijas, que tratan de compaginar trabajo y cuidado de los hijos, representa su ideal. Para la mayoría de ellas no había más opciones, era una cosa o la otra. Trabajar era incompatible con tener hijos.

"El ideal si yo hubiera tenido esa opción, es compatibilizar ambas cosas. Moderadora: ¿Por qué? Porque me habría sentido satisfecha de sacar a mi familia adelante a la vez que yo me realizaba como persona (...) si no me hubiera visto obligada a elegir habría sido el ideal" (Esperanza).

No obstante, el motivo por el que las madres recomiendan a sus hijas que no deben dejar de trabajar tiene como fin más la seguridad de sus propias hijas que la idea de que es bueno para sus nietos que sus madres se ocupen de ellos; siguen actuando como madres cuidadoras que velan por el interés de sus hijas. El principal argumento que utilizan las madres es el de la vulnerabilidad que tiene una mujer cuando depende completamente de la pareja para vivir.

\section{MADRES:}

"Ahora pienso fríamente, tuve un marido maravilloso, pero ¿y si me hubiera ido mal? ¿Cómo me hubiese quedado? Te la juegas, hay que ser realista” (Ma José).

"Ahora le digo a mi hija jni se te ocurra dejar el trabajo!” (Estrella).

HIJAS:

"A mí (mi madre) me dice que hago bien en reducir la jornada, en estar con mis hijos, pero que no pierda el trabajo, que hoy en día nunca se sabe. (...) Ven como la gente se separa, los problemas que hay... Al final quieren protegerte $y$ darte a entender ese es tu sustento" (Ana).

"Mi madre me dice que no dependa nunca de nada ni de nadie, ni de un trabajo ni de un hombre" (Teresa).

Las hijas han adoptado este modelo, pero, sin embargo, no es tan ideal como las madres lo imaginan. Viven esta situación con la sensación de que al final no consiguen ni una cosa ni otra, de que se quedan a medias.

\section{"Ellas no iban tan estresadas" (Marta).}


Del análisis de los relatos de las madres y de las hijas observamos como el ideal de madre que tienen unas y otras está lógicamente influido por el contexto cultural y social de la época en la que viven (nivel institucional del modelo) y tiende a confluir en el momento presente, dado que las madres han pasado por un proceso de adaptación. En este sentido, la mayoría de las madres tratan de inculcar en sus hijas un modelo más acorde a la sociedad actual que a la que ellas vivieron y se acercan más al modelo de madre conciliadora o cuidadora principal que al de cuidadora exclusiva que muchas siguieron. Todas ellas creen que es responsabilidad de la madre estar con sus hijos en la etapa infantil, incluso algunas más allá, en la adolescencia, y luego, cuando son más mayores, creen que el cuidado podría ser compartido entre padres y madres. Para ello ven natural que las hijas sean las que tomen en exclusiva los permisos de maternidad y que, posteriormente, se reduzcan la jornada. Todas consideran que sus hijas no deben dejar el empleo por completo, aunque reconocen que esto supone tanto dificultades logísticas para sus hijas, en cuyo caso suelen ser ellas las que las ayudan, tanto como dificultades para prosperar profesionalmente.

"Yo igual soy un poco antigua, pero pienso que un bebé recién nacido necesita más a su madre que a su padre. Luego ya no, cuando son mayores ya da igual el padre que la madre. pero al principio si, los primeros años (...) todo el tiempo que se pueda. No sé cuánto... Yo si hubieran tenido que dejar al hijo recién nacido habría sido un trauma, pero a lo mejor cuando tienen un poco más edad no" (Esperanza)

"De pequeñitos (mejor la madre), luego ya daría igual, me refiero hasta unos 8 meses" (Silvia)

"Mi hija dejó de trabajar porque ya son cuatro niñas y necesitan a su madre, o que este yo, yo voy a ayudarle" (Maite)

Las hijas reconocen y valoran los aspectos positivos que para la mayoría de ellas supuso la estrategia seguida por sus madres durante su infancia, experiencia que deja una importante huella e influye en la construcción de su modelo ideal, como vimos en el marco teórico, pero no es el único elemento del modelo, el contexto cultural va a ser igualmente determinante en el modelo que las hijas construyan.

"Yo pienso que en el momento en el que tienes hijos, tienes que pensar primero en lo mejor para tus hijos, y después para ellas, o que no tengan hijos, que también es una opción, pero si los tienes tienen que pensar en lo que es mejor para sus hijos y luego para ellas" (Esperanza) MOD ¿Estáis de acuerdo? ¿habéis inculcado eso a vuestras hijas? "Si" (la mayoría asiente). 


\subsection{4. ¿Se hereda el modelo?}

Consideraremos que el modelo de maternidad se hereda cuando madre e hijas muestran similares ideales y prácticas. Utilizaremos la tipología de González y Jurado (2015) para clasificar a las madres y a las hijas y observar así sus ideales y sus prácticas en relación a la maternidad.

De los siete casos analizados, se observa como en cinco casos el discurso de madres e hijas es similar mientras que en dos de ellos se observa una tendencia 'bumerang', una cierta compensación sobre lo que han vivido.

Entre los cinco casos en los que se replica el modelo encontramos diferentes tipos de mujeres y familias, mujeres más instruidas y menos, y mujeres con una ideología más liberal y otras más conservadoras.

Dos casos, $\mathrm{M}^{\mathrm{a}}$ Luisa y $\mathrm{M}^{\mathrm{a}}$ José, con sus hijas Marta y Ana, presentan bastantes similitudes. $\mathrm{M}^{\mathrm{a}}$ Luisa y $\mathrm{M}^{\mathrm{a}}$ José ambas dejan de trabajar al casarse y se dedican a cuidar de sus hijos, incluso cuidan de la suegra. Ambas, llegado el momento, tuvieron que ayudar temporalmente en el negocio del marido, por lo que no cobraron ningún sueldo, ni se les pagó seguridad social. Su modelo familiar es el de reparto claro de las responsabilidades del hogar según género, madre cuidadora y padre sustentador y, según la tipología de González y Jurado serían "madres cuidadoras convencidas" (González y Jurado, 2015: 92). Ambas tienen un discurso tradicional sobre la maternidad, en el sentido de pensar que las madres deben ser las responsables principales del cuidado de los hijos porque es lo más natural, aunque reconocen que hoy en día si se puede no se debería dejar el empleo por los riesgos que supone, y que no es justo, que sus hijas, que tienen una formación se hayan visto perjudicadas en sus profesiones por dedicarse a cuidar a sus hijos.

"Los hijos la necesitan, llegar del colegio, hasta las 7 de la tarde no vienen ellos y encontrarla ahí. Yo la veo (a $\mathrm{m}$ i hija) como yo fui e incluso más madraza, trabaja demasiado. (Mi hija) trabaja en la oficina y se ha buscado otro trabajo. Ni tanto ni tan calvo." ( $M^{a}$ Luisa)

"Era dejarlos todo el día con una persona desconocida o cuidar y disfrutar de ellos. (...) quería disfrutar de ella (su hija) al máximo (...) Pues a mí me da pena (que se haya visto relegada en el trabajo por la reducción de jornada) ... porque todos mis esfuerzos por formarla (...) pienso que no va a poder ejercer nunca, ni premiar su esfuerzo, ni la ilusión de sus padres porque sea alguien mejor" (Masé)

Sus hijas, Marta y Ana, licenciadas superiores, con aspiraciones profesionales, pero que han supeditado su carrera al cuidado de los hijos muestran un discurso alineado con sus madres y una gran satisfacción en cómo fueron sus infancias, en el cuidado y la atención que recibieron de sus madres. El caso de Ana podría clasificarse por una parte, como "madre cuidadora convencida" (González y Jurado, 2015:92) ya que ve con naturalidad el reparto desigual de las tareas (sic) y vive la maternidad de forma positiva, sin ambivalencias. Aunque, por otra, se acerca al modelo de "madre cuidadora corresponsable" ya que su pareja, funcionario, tiene cierta facilidad de horarios para ayudar en casa. 
"Tengo muy claro que mis prioridades son mi familia lo primero y el trabajo es un complemento. La reducción de jornada sí, porque mis hijos son míos, y no de mi madre ni de mi suegra, e intento organizarme al máximo para apañarnos (...) A mí me dice (mi madre) que hago bien en reducir jornada, en estar con mis hijos, pero que no pierda en trabajo, que hoy en día nunca se sabe. (...) $Y$ me planteo en el futuro es cambiar de departamento, tengo un buen convenio, soy estatutaria. Tengo unas buenas condiciones. Mi marido es funcionario también y la prioridad que tenemos es nuestra familia" (Ana)

Sin embargo, Marta transmite una gran frustración por el castigo al que ha sido sometida en su empresa por haber reducido la jornada, lo que compensa con otras actividades por su cuenta, más creativas, que le satisfacen más, y justifica la poca ayuda del padre por su dependencia del trabajo al tener un cargo directivo, de lo que se queja. Marta podría considerarse como una "madre cuidadora principal ambivalente" (González y Jurado, 2015:94) ya que vive con bastante tensión su papel de madre y empleada respecto a lo que le hubiera gustado. Le gustaría recuperar el estatus que tenía en la empresa antes de reducirse la jornada, pero con los horarios que la empresa tiene lo considera imposible.

"A veces lo ves y piensas lo duro que es, a pesar de no tener unas aspiraciones laborales muy grandes (...). No lo cambiaria, pero sale duro. (...) Con los horarios que tiene la empresa. No podía tener un niño en la guardería hasta las 19,30 o las 20,00h. (Marta)

El caso de Maite y su hija Raquel podría considerarse dentro del grupo de madres cuidadoras convencidas como el anterior. No muestran demasiadas ambivalencias por las contradicciones entre el trabajo y la maternidad, ambas han trabajado por necesidades económicas más que por una orientación profesional fuerte y han podido compaginar trabajo y cuidados gracias a la ayuda de los padres y de la familia extensa, en concreto de los abuelos.

"Mi hija dejó de trabajar porque ya son cuatro niñas y necesitan a su madre, o que esté yo, yo voy a ayudarle. (...) Pero como el padre cobra más... (...). Yo hice eso en su día porque es lo que me tocó." (Maite).

"Después de la primera hija me di cuenta de que no iba a sacarlo jamás (el título de técnico de enfermería) ... Ganaba menos en el trabajo por ser auxiliar. Ahora ya no hago planes. Antes bueno tenía la carrera esta... y que luego es menos dinero... Me lo decían... pero bueno a disfrutar de las niñas. Pero bueno, es lo que decidí en su momento y he disfrutado mucho de ellos. Con la cuarta me di cuenta de que la reducción de jornada me hacía falta. Mi marido tiene jornada completa, es profesor, y era imposible. Y dije ya está. Yo he disfrutado mucho de las niñas." (Raquel).

Estrella y Reina, madres de Teresa y María, son mujeres con un discurso menos tradicional. Se clasificarían como "madres cuidadoras principales ambivalentes" (González y Jurado, 2015:94). Ambas tenían una orientación profesional fuerte que se vio afectada por la maternidad o el trabajo del marido, especialmente en el caso de Estrella, y se mantiene hoy en día. Tratan de inculcar en sus 
hijas un modelo ideal que, aunque en la práctica ellas no pudieron vivir, sus hijas si han conseguido.".

"Yo si volviera a nacer, desde luego mi trabajo no lo dejaba por nada del mundo. Lo que he aprendido a lo largo de mi vida, no es que mis hijos no me valoren, me valoran como persona, pero cuando son pequeños es un orgullo que las madres trabajen, es como decir mi madre es lista y vale, por decirlo de alguna manera, y la que está en casa no vale (...). ¡Ahora presiono a mi hija "ni se te ocurra dejar el trabajo!" (Estrella)

"Yo sí, lo tenía clarísimo que quería seguir trabajando. (...) Mi hija sigue siendo muy inquieta: apuntándose a cursos, formándose... Creo que su carrera continuará después de su maternidad. Aunque sea a media jornada seguirá toda la vida. Yo no recomiendo alargar mucho la reducción de jornada por este motivo, a mí me pasa que no la recomiendo porque me faltan años de cotización, y como no encuentro trabajo me va a quedar una pensión ridícula. (Reina).

Teresa y María tienen también una fuerte orientación al trabajo. Se sienten privilegiadas porque trabajan como enfermeras en la sanidad pública lo que supone una garantía y una seguridad laboral. Podrían ser clasificadas como "madres cuidadoras conciliadoras" (González y Jurado, 2015:94) ya que su profesión les permite un buen equilibrio entre las exigencias de ser una buena madre y las de buena trabajadora. La sanidad pública les permite seguir formándose y adaptar sus horarios, cuentan con bastante apoyo de los padres y de sus madres para lidiar con los problemas de los turnos, a veces nocturnos. Como hijas, se muestran satisfechas del cuidado que recibieron de sus madres y a la vez manifiestan una gran admiración por el esfuerzo y sacrificio que éstas tuvieron que hacer.

"Me cogí la reducción de jornada por necesidades de lactancia y continúo hoy por el trabajo de Antonio, porque ahora él pasa mucho menos tiempo en casa. El trabajo en casa lo hacemos un poco entre los dos (...) me parece una gran suerte el funcionamiento de este tipo de procesos en la empresa pública. Yo también, lo veo como una ventana. Ahora vivo esto porque es lo que toca y porque es irrepetible, y luego ya volveré" (María).

"Yo tenía claro que no quería dejar mi trabajo porque luego volver no es fácil. (...) No hay un entorno favorable para la maternidad, está de moda la conciliación, pero es mentira, no existe y eso que yo estoy en lo ideal... pero parece que los hijos estorban en las empresas, en la de mi chico no pudo cogerse los días [de permiso de paternidad] porque está mal visto y es tanta la presión en su entorno... mira a ver si cogen represalias por los quince días" (Teresa).

"Mi madre, era contable, se pidió medía jornada y yo creo que al final perdió un poco el tren, porque acabó trabajando en la empresa de mi padre. Si hubiera trabajando en otro sitio habría tenido mucho más éxito. (...) aunque ha terminado aprendiendo todo" (María)

"Mi madre dice que se arrepiente de las decisiones que ha tomado. Mi madre tenía muy buen puesto de trabajo, un puesto que en un tiempo actual habría obligado a cogerse la reducción a mi padre ....En su día le tocó a ella, ya que mi padre viajaba por trabajo y además era algo impuesto por la época, y creo que ella habría continuado con su carrera" (Teresa). 
"A mí, mi madre siempre me dice que no dependa nunca ni de nada ni de nadie: ni de un hombre, ni de un trabajo, que no dependa nunca de nadie para poder vivir, que es le ha pasado a ella (Teresa), Exacto, nos ven más independientes". (Marta)

Hasta aquí serían los cinco casos en los que el ideal sobre la maternidad se hereda de madres a hijas y en unos casos (los tres primeros) supone la herencia también de las prácticas, mientras que en otros (los dos últimos) no se llegan a heredar las prácticas dado que las madres consiguen que la ambivalencia que vivieron no se replique en el caso de sus hijas, madres cuidadoras conciliadoras.

A continuación, se presentan dos casos en los que se podría decir que el modelo de las hijas tiende a compensar lo que éstas consideran unas estrategias muy extremas en sus madres, en una u otra dirección. Son los casos de Esperanza y su hija Irene y de Silvia y su hija Lara.

Esperanza es la madre más joven (55 años) entre las madres y tiene seis hijos. Es una "madre cuidadora convencida" (González y Jurado, 2015:92), que se ha dedicado enteramente al cuidado de sus hijos y de sus padres mayores. Es una madre entregada que se queja del poco reconocimiento que tiene su función de cuidadora en la sociedad y que ve en su hija un cambio de mentalidad que no le llega a parecer bien. Piensa que su hija (Irene) no es tan entregada como ella y ve en ella un cierto egoísmo que extiende a las nuevas generaciones. El caso de Irene es algo complejo ya que actualmente en su relato observamos numerosas manifestaciones de felicidad por poder cuidar de su único hijo y poder disfrutar de su maternidad, pero a la vez muestra una fuerte orientación al empleo y una gran frustración por haber tenido que frenar su carrera profesional, que desea retomar en breve plenamente, aprovechando su reducción de jornada para formarse. Por otra parte, se muestra muy contraria al ideal de maternidad (incluso de estilo de vida) de su madre y menciona expresamente que no lo comparte. Podría considerarse un caso de madre cuidadora principal ambivalente por las circunstancias con las que se ha encontrado, pero una madre cuidadora corresponsable por ideales. Este sería, por tanto, un caso de ruptura del modelo de maternidad tanto en las prácticas como en los ideales.

"Yo pienso que en el momento en el que tienes hijos, tienes que pensar primero en lo mejor para tus hijos, y después para ellas, o que no tengan hijos, que también es una opción, pero si los tienes tienen que pensar en lo que es mejor para sus hijos y luego para ellas" (Esperanza).

"(Mi madre) un poco sometida si estaba (...) Ahora nosotros decidimos todo de manera consensuada, hay comunicación, los dos tenemos autoridad y criterio (...) Mi marido hace exactamente lo mismo que yo, y mi madre alucina (...) Mi madre me ve como que no doy el 100\%. Sí, mi hijo... pero (dice) que yo cuento mucho, que podría hacer más cosas de las que hago, que me podría sacrificar más, que tengo también tiempo para mi... Sé que en el fondo no lo ve, no le termina de cuadrar, el momento de madre abnegada no lo ve. (...) Ella piensa que si redujese más mi jornada para estar con mis hijos sería mejor, piensan que es lo que debería de hacer, más que llevarle pronto al desayuno en el cole. ¿Por qué no entras a las 10? Siempre me aconseja relegar más mi 
trabajo por mis hijos (...) Me veo todavía muy joven, mi hijo se está haciendo mayor. Creo que tengo una segunda oportunidad a ver si me subo al tren. (...) El sueldo también es un factor. El que gana más es el que trabaja más horas. Si yo ganara más que él seguramente él tendría la reducción de jornada. No es una cuestión de madre o padre. Es un tema de economía familiar. (...) Creo que mi sitio es trabajar por mi cuenta y sí me he interesado en hacer cursos de páginas web (...) y despegar, pero cuando Carlos tenga 12 años, (...) seria auto gestionarme yo" (Irene).

Por otra parte, nos encontramos con un caso en el que la madre (Silvia) ha tenido una fuerte orientación al trabajo y una sola hija que se queja de haber recibido poca atención de su madre y que, al tener sus propios hijos, dice abiertamente no querer repetir tal cual la estrategia de su madre. Este caso, el de Silvia y Lara es un claro ejemplo de ruptura con el ideal de modelo materno y ambas lo expresan abiertamente. Silvia ha tenido una sola hija que ha estado atendida con ayuda externa para poder compaginar su carrera profesional con cuidar de su hija. Es una mujer que se siente por una parte muy orgullosa de lo que ha conseguido profesionalmente, pero, por otra, algo arrepentida como madre porque, según ella misma dice, su hija se lo reprocha. Lara, por su parte, interrumpe una carrera profesional en el extranjero con un buen potencial ante la llegada del primer hijo, que desea tener en España y así aprovecha además para ayudar en la empresa familiar que atravesaba por ciertas dificultades. Aunque esto, según ella misma cuenta, supone un importante freno para su carrera se muestra convencida de que cuidar a su hijo en este momento es prioritario en su vida y por eso ha reducido su jornada. Lara podría clasificarse como "madre cuidadora convencida" (González y Jurado, 2015:92) ya que el trabajo para ella, aunque es importante, ha pasado claramente a un segundo plano.

"Para mí el ideal es la situación que vivo ahora. Todo está cubierto. Mi marido no llega a casa muy tarde, llega a las 6, y tenemos tiempo para hacer cosas juntos, habiendo yo ya recogido al niño, merendado, ido al parque. (...) ...tienes tus cartas, a lo mejor no son una pareja de ases, pero ...A veces pienso en el regreso a la jornada completa y debe ser tan complicado encajarlo... Después de tanto tiempo, no lo pienso mucho, pero la verdad, tu hijo con 12 años y tú llegando a casa a las 8 de la tarde. No sé qué pasará entonces. (...) Mi madre y mi padre han estado a la par, han sido muy modernos" (Lara).

\section{CONCLUSIONES}

Por lo tanto ¿podemos decir que, en los casos analizados, el modelo materno influye en el ideal de maternidad de sus hijas? En caso afirmativo, es decir, si se constata una influencia, ¿cómo se construye el modelo de las hijas?

Retomando la hipótesis de partida y en los casos analizados en este trabajo se puede afirmar que el modelo de maternidad de las madres ha influido en 
las hijas, en una dirección o en otra, directa o indirectamente. Más allá de los aspectos culturales y sociales y de su evolución en el tiempo, por una parte, las madres han influido, obviamente y de forma implícita, en primer lugar, como madres que fueron. El recuerdo de la niñez, con la presencia o no de la madre en casa, y la valoración que las hijas hacen ahora, desde la madurez, de la experiencia vivida por sus madres, de las circunstancias que vivieron y de las decisiones que tomaron, son factores que forman parte del proceso de socialización y de construcción de la identidad personal de las hijas. Pero, por otra parte, y de manera explícita, muchas madres manifiestan aconsejar y tratar de influir en sus hijas. Por lo tanto, la influencia tanto implícita como explícita, se constata en este trabajo. Pero, una vez confirmada la hipótesis de la influencia, las decisiones de las hijas respecto a la estrategia a adoptar pueden seguir o no el ejemplo materno. ¿De qué depende que esto sea así? En los casos analizados en este trabajo observamos que el hecho de que esta herencia siga la misma dirección o siga una dirección opuesta depende de dos factores relacionados con cómo viven y qué piensan las hijas respecto a la estrategia seguida por sus madres.

Un primer factor sería la medida en que la estrategia seguida por las madres en su época no se haya distanciado sustancialmente de las costumbres y hábitos de la época contemporánea, cuando las hijas son madres, es decir, que no haya sido una estrategia que se considere hoy completamente inasumible por sus hijas incluso acometiendo algunas adaptaciones. A este factor lo podemos denominar la contemporaneidad de la estrategia seguida por las madres. En el eje generado por este factor tendríamos dos extremos: en un extremo madres que dejaron su empleo, que se han dedicado en exclusiva al cuidado de los hijos, que consideramos menos contemporáneo o más tradicional y, en el otro extremo, lo que considero más contemporáneo, madres con una gran dedicación al empleo que trataron de conciliar con los cuidados. Se podrían situar a lo largo del eje los casos de madres que han trabajado a tiempo parcial o ayudando en el negocio familiar.

Un segundo factor sería que la estrategia seguida por sus madres haya significado una vivencia satisfactoria o insatisfactoria para las hijas en su infancia según se desprende de sus discursos.

A partir de estos dos factores se podrían posicionar los casos analizados en un plano cartesiano en el que en el eje horizontal se situaría la satisfacción/ insatisfacción con la estrategia seguida por las madres y en el eje vertical la contemporaneidad/tradicionalidad (desde la perspectiva actual) de la misma. 


\section{GRAFICO 1 Factores relacionados con la herencia del modelo materno}

\section{CONTEMPORÁNEO}

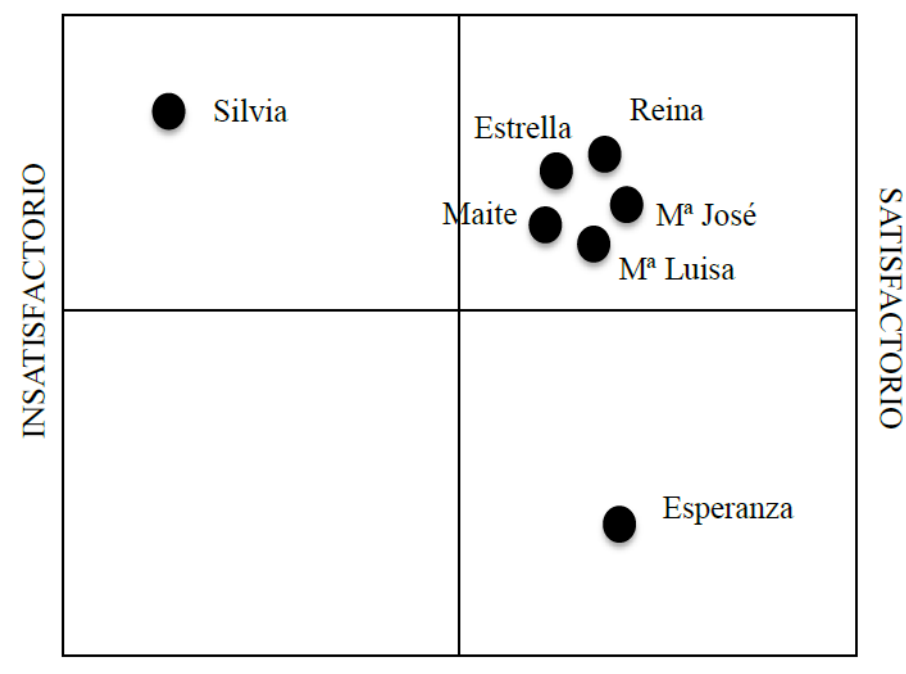

TRADICIONAL

Nota: En el gráfico se representan a las madres desde la perspectiva de las hijas

Por un lado, en el cuadrante de la satisfacción y la contemporaneidad se situarían cinco de los siete casos. Resumiendo, sus principales características, cuya descripción se ha realizado anteriormente, en ellos, la estrategia de las madres se considera contemporánea, en el sentido de corresponderse con el modelo más común actualmente de padre sustentador principal y madre cuidadora principal, ya que se trata de mujeres que han trabajado y tratado de conciliar el cuidado de los hijos con la actividad laboral. El modelo ideal de estas mujeres, con algunas variaciones de adaptación al entorno actual, es fácilmente asumible por sus hijas. Respecto al plano de la satisfacción, las hijas expresan satisfacción como hijas, con la estrategia seguida por sus madres.

Por otro lado, tendríamos dos casos en diferentes cuadrantes. Un caso, Silvia, se sitúa en el cuadrante de la insatisfacción, explícitamente manifestada por la hija, Lara, y la contemporaneidad, ya que la madre se sitúa lejos del papel de cuidadora exclusiva y, con una fuerte centralidad laboral, está más cerca del modelo de madre corresponsable.

En el extremo contrario, en el de la satisfacción y tradicionalidad tenemos el otro caso, Esperanza, una mujer cuya estrategia se centra en exclusiva en el cuidado de los hijos y los mayores, sin actividad laboral. Su hija se distancia de ese modelo claramente y aspira a un ideal diferente, aunque en el momento del estudio no lo puede llevar a la práctica. 
Observamos como en el cuadrante de la contemporaneidad y la satisfacción, se localizan los casos en los que las hijas comparten el ideal y en el caso en el que no comparten las prácticas es porque las madres les inculcan un cambio de modelo, es decir, se podría decir que el modelo se traslada de madres a hijas. Sin embargo, en los otros dos casos, las hijas rompen con el modelo seguido por sus madres.

Por lo tanto podríamos concluir que en los casos analizados en este trabajo la hipótesis de la que se partía, es decir, que las madres ejercen una gran influencia en la estrategia seguida por sus hijas, se confirma, y que para que las hijas hereden el modelo ideal de sus madres tal cual es necesario por una parte que la estrategia seguida por sus madres haya sido satisfactoria para la hija en su infancia y, por otra, que se considere social y culturalmente acorde al pensamiento del momento, que, según el CIS (CIS, 2012) es el de «una familia en la que los dos miembros de la pareja tienen un trabajo remunerado con parecida dedicación y ambos se reparten las tareas del hogar y el cuidado de los hijos/as, si los hay» (72\%), aunque cuando la madre trabaja y tiene un hijo menor de 14 años, el porcentaje de madres que apoya este modelo desciende desde el $80 \%$ al $66 \%$, porque se considera que hay más madres que apoyan el modelo en el que "uno de los miembros de la pareja tiene un trabajo remunerado con menor dedicación". En cualquier caso, es minoritario el modelo de padres sustentador y madre ama de casa.

\section{BIBLIOGRAFÍA}

ABRIL, P, JURADO, T, y MONFERRER, JM (2015): Paternidades en construcción en GONZÁLEZ, MJ, y JURADO, T (2015): Padres y madres corresponsables. Una utopía real, Madrid, Los libros de la catarata.

ALBERDI, I, ESCARIO, P Y MATAS, N (2000): Las mujeres jóvenes en España, Barcelona, Fundación La Caixa.

BITTMAN, M, ENGLAND, P, SAYER, L, FOLBE, N AND MATHESON, G, (2003); "When does gender trump money? Bargaining and time in household work", disponible en http://ro.uow.edu.au/cgi/viewcontent.cgi?article=1088\&context=arts papers [Consulta: 21-11-17]

BOTÍA, C, DOMÍNGUEZ, M Y JURADO, T (2015): "Maternidad corresponsable", en GONZÁLEZ, MJ y JURADO, T (2015): Padres y madres corresponsables. Una utopía real, Madrid, Los libros de la catarata. pp. 56-99.

CALLEJO, J (2001): El grupo de discusión: introducción a una práctica de investigación, Barcelona, Editorial Ariel.

CANTÓN, O, CEBRIÁN, I Y MORENO, G (2015): “Género y pobreza”, en CASTAÑO, (2015): Las mujeres en la gran recesión, Madrid, Ediciones Cátedra , pp. 121-168.

CASTAÑO, C (2015): Las mujeres en la gran recesión, Madrid, Ediciones Cátedra.

CASTAÑO, C (2015): "Introducción", en CASTAÑO, C (2015): Las mujeres en la gran recesión, Madrid, Ediciones Cátedra, pp. 7-49.

CIS, (2012): Estudio 2973. Conocimiento sobre la realidad sociopolítica y económica, disponible en http://www.analisis.cis.es/cisdb.jsp [Consulta: 15-07-17] 
CORBETTA, P (2003): Metodología y técnicas de investigación social, Madrid: McGraw-Hill/interamericana de España.

DEAUX, K, y MAJOR, B (1990): “A Social-Psychological Model of gender" en RISMAN, By DAVIS, G, (2013): "From Sex Roles to Gender Structure", disponible en http://journals.sagepub.com/doi/abs/10.1177/0011392113479315 [Consultado 15-07-17]

GARRIDO, L (1998): "La ocupabilidad en la familia", en Papeles de Economía Española, 77,Funcas, pp 41-90.

GONZÁLEZ, MJ y JURADO, T (2015): Padres y madres corresponsables. Una utopía real, Madrid, Los libros de la catarata.

GONZÁLEZ, MJ (2015): "Padres primerizos en tiempos de crisis", en GONZÁLEZ, MJ y JURADO, T (2015): Padres y madres corresponsables. Una utopía real, Madrid, Los libros de la catarata. pp. 31-55.

HAYS, S (1998): Las contradicciones culturales de la maternidad, Barcelona, Paidós contextos.

LLORENTE, R, IGLESIAS, C y DUEÑAS, D (2015): "Mujer, mercado de trabajo y crisis económica”, en CASTAÑO, C (2015): Las mujeres en la gran recesión, Madrid, Ediciones Cátedra, pp. 51-86.

LOPATA, HZ y THORNE, B (1978): “On the term sex roles” en RISMAN, B J. Y DAVIS, G (2013): "From Sex Roles to Gender Structure" disponible en http:// journals.sagepub.com/doi/abs/10.1177/0011392113479315 [Consultado 15-07-17].

PFAU-EFFINGER, B (1998): "Gender cultures and gender arrangement: a theoretical framework for cross-national gender research" en CASTAÑO, C (2015): "Las mujeres en la gran recesión”, Madrid: Ediciones Cátedra

RICOEUR, P (2006): Ideología y utopía, Barcelona, Editorial Gedisa.

RISMAN, B J. y DAVIS, G (2013): From Sex Roles to Gender Structure, Current Sociology Review, disponible en http://journals.sagepub.com/doi/ abs/10.1177/0011392113479315 [Consultado 15-07-17]

RITZER, G (2001): Teoría Sociológica clásica, Madrid, McGraw Hill.

THOMPSON, L (1991): "Family Work: Women's Sense of Fairness", Journal of Family Issues, Disponible en http://journals.sagepub.com/doi/ abs/10.1177/019251391012002003 [Consultado 15-07-17]

TOBÍO, C y FERNÁNDEZ, JA (2015): "El déficit de cuidado ante la crisis", CASTAÑ , C (2015): Las mujeres en la gran recesión, Madrid, Ediciones Cátedra, pp. 201-238. 
